\title{
In-Video Reuse of Discussion Threads in MOOCs
}

\author{
Qian Zhao \\ Computer Science \\ University of Minnesota \\ Minneapolis, United States \\ qian@cs.umn.edu
}

\author{
Sashank Varma \\ Educational Psychology \\ University of Minnesota \\ Minneapolis, United States \\ sashank@umn.edu
}

\author{
Joseph A. Konstan \\ Computer Science \\ University of Minnesota \\ Minneapolis, United States \\ konstan@cs.umn.edu
}

\begin{abstract}
In MOOCs, both instructors and students invest substantial effort into discussion forums. However, those discussions are abandoned when instructors start a new session in session-based courses. In an observational field study through a popular online Coursera course, we evaluate an approach that directly embeds high-value past discussion threads into future lecture videos to reuse them. Survey feedback shows that this approach can be useful to a large proportion of learners. We find that instructor involvement increases learners' chance of reading the threads, reduces learners' negative reactions, but is not associated with more perceived usefulness. Learners perceive enhancing threads embedded in the middle of videos less enhancing but more explanatory compared with at the end. Embedding explanatory threads at the end is rated less distracting and more helpful to understand the video compared with in the middle, right after the related content is lectured.
\end{abstract}

\section{Author Keywords \\ MOOCs; Computer Supported Collaborative Learning.}

\section{INTRODUCTION}

In Massively Open Online courses (MOOCs), discussion forums are the basic media for students and instructors to interact with each other. Discussion threads enable students to socially co-construct knowledge with peers, which prior research shows is essential for learning [7]. Both instructors and learners invest substantial effort into these discussions. We believe that these highly-voted threads are potentially re-usable learning assets that can be incorporated into future sessions of courses to address known questions and stimulate deeper learning. However, these discussions are not generally indexed by search engines because of the signing-up barrier and because of proprietary concerns. Transferring the knowledge in these discussions to future course offerings is especially difficult for session-based courses, where sequential cohorts of learners attend, leaving previously offered sessions almost abandoned.

Permission to make digital or hard copies of all or part of this work for personal or classroom use is granted without fee provided that copies are not made or distributed for profit or commercial advantage and that copies bear this notice and the full citation on the first page. Copyrights for components of this work owned by others than ACM must be honored. Abstracting with credit is permitted. To copy otherwise, or republish, to post on servers or to redistribute to lists, requires prior specific permission and/or a fee. Request permissions fromPermissions@acm.org.

L@S 2017, April 20-21, 2017, Cambridge, MA, USA

(C) 2017 ACM. ISBN 978-1-4503-4450-0/17/04 \$15.00

DOI: http://dx.doi.org/10.1145/3051457.3053972
In this paper, we explore directly embedding high-value past discussion threads into the lecture videos for future course sessions. We think this approach might be useful because of two reasons. First, previous research in learning and education shows that in-classroom question-and-answer interactions between the instructor and students, or among students, are important to learning [4]. The proposed approach might be able to mimic that interactive learning experience to some extent. Second, in-time help or feedback might reduce learners' cognitive load - either extrinsic load because of unclear instruction or intrinsic load inherent in the difficulty of the content to be learned [3]. Previous research in intelligent tutoring systems also shows that the best tutoring style is one which gives immediate feedback [1].

We are facing two challenges in implementing the embedding approach in the current MOOCs. First, it is not obvious how to decide which threads to use. Unlike annotated discussions studied in previous research [2], these threads are wide open discussions and there are no direct links between the threads and videos. Second, the embedded threads are not designed together with the lecture videos. As a result, embedding threads into videos might interrupt the flow of instruction. Given (1) high quality threads that are (2) well-matched with lecture videos, we still need to decide on a time point to display the thread. Therefore, we adopted the Wizard of $\mathrm{Oz}$ [5] approach by manually selecting high-value threads and matching them with time points in the lecture videos. We answer two research questions in our study.

RQ1: Is directly embedding peer discussion threads into lecture videos useful to learners?

RQ2: How do different design factors for embedding threads affect learner reactions? We identify three design factors (two involving thread selection, one involving the embedding itself) that are particularly interesting and generalizable to different courses:

- instructor-involvement (whether instructors replied to the thread)

- explanatory vs. enhancing thread type (whether the thread explains a lecture point or provides enhancing content beyond the lecture)

- timing of the interruption (at a relevant point in the middle vs. at the end of the lecture). 


\section{RESEARCH DESIGN}

We conduct an observational field study in an online course Introduction to Recommender Systems (one of the authors is the instructor of this course) offered through Coursera. Our design depends on utilizing threads as they naturally occurred to maintain ecological validity. We selected 24 threads from the discussion forums in the session-based offering of the course and embedded them into 24 lecture videos (one thread for each video) in the on-demand version in a pop-up format similarly to in-video quizzes as shown in Figure $1^{1}$. The threads were selected to balance conditions of instructor-involvement (yes vs. no) and thread type (explanatory vs. enhancing) and then assigned to one timing condition (in the middle vs. at the end of the lecture video). In total, three threads were present in each of the eight $(2 \times 2 \times 2)$ conditions. The study followed a withinsubjects design: if a learner taking the on-demand offering went through all the lecture videos, then they saw three discussion threads in each of the eight conditions.

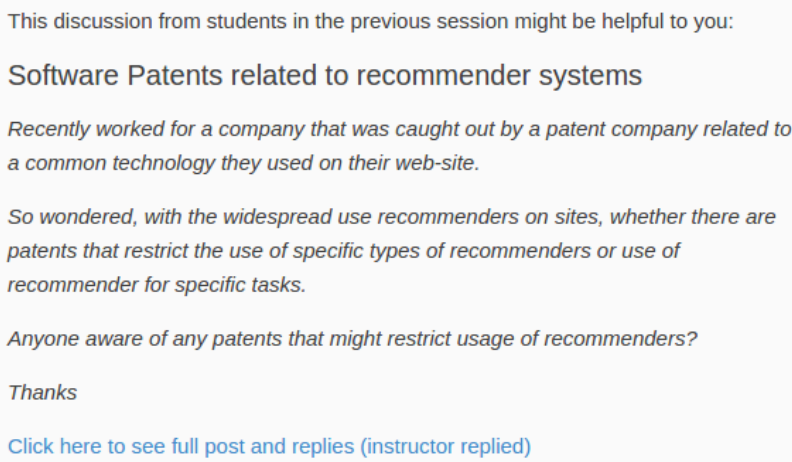

Figure 1. The in-video quiz pop-up interface in Coursera with our selected discussion thread.

We used three types of measurements in this study. The first one measures engagement with the lecture video. Specifically, we used the metric of Completion Rate defined as the percentage of learners watching to the end of a lecture video out of those who started watching it. We compared the completion rates of two time periods: three weeks before and three weeks after the embedded threads were taken off. Time can be a confounding factor here, but since there are no other changes to this on-demand course during the two periods, we think that this is a reasonable comparison. The second measurement is Click-Through Rate (CTR), i.e. the probability of learners clicking the displayed thread link given that they see the question text of the thread.

In addition, we directly embedded a survey into the video lecture associated with each thread and displayed it immediately after the thread pop-up was dismissed. We show learners six options of which they could select zero or more, grouped in one survey pop-up as shown in Table 1.

\footnotetext{
${ }^{1}$ See http://tinyurl.com/jo74fkc for the selected threads.
}

\begin{tabular}{|c|c|}
\hline Option Label & Full Text in the Survey \\
\hline helpful to video & $\begin{array}{c}\text { I found it helpful to me in understanding } \\
\text { the video }\end{array}$ \\
\hline useful extra & $\begin{array}{c}\text { I found it useful extra information beyond } \\
\text { the video }\end{array}$ \\
\hline unwanted & I did not need/want this \\
\hline distracting & $\begin{array}{c}\text { Interrupting me with the discussion was } \\
\text { distracting }\end{array}$ \\
\hline didn't read & I did not read the discussion thread \\
\hline $\begin{array}{l}\text { helpful but } \\
\text { distracting }\end{array}$ & $\begin{array}{c}\text { I found it interesting or useful, but would } \\
\text { prefer not as an interruption in the video }\end{array}$ \\
\hline
\end{tabular}

Table 1. The survey options to collect feedback from learners. The order of the options is shuffled randomly each time the survey is displayed.

The order of the options was shuffled randomly each time. After making their choices, learners were shown the responses of their peers aggregated in a poll. By considering all possible cases of checking the six options, we classified each survey response into six exclusive categories: unknown (checking none of the six options), strong negative (only checking "unwanted" or "distracting"), strong positive (only checking "helpful to video" or "useful extra"), mixed (only checking "helpful but distracting" or "didn't read"; Otherwise, checking both strong positive and strong negative), weak negative (checking both strong negative and mixed but not strong positive), weak positive (checking both strong positive and mixed but not strong negative).

\section{RESULTS}

The study lasted from August 16, 2015 until March 15, 2016. During the study period, 4,593 learners saw the embedded threads. In total, the thread pop-ups (Figure 1) were displayed 21,164 times and the survey pop-ups were displayed 16,953 times. We collected 15,407 survey responses in total.

$R Q 1$. First of all, we analyze the effect of embedding discussion threads in the middle of lecture videos on completion rate. During the two periods (three weeks before and after the threads were taken off), 1227 learners started watching the relevant lecture videos 3666 times and completed watching them 2190 times (i.e. completion rate $=59.7 \%$ ). These data only include in-the-middle embedding conditions because in at-the-end condition learners had by definition already completed the lecture video. We employ a mixed-effect logistic regression analysis, in which we model having embedded threads as a fixed effect and model learners as a random intercept. The fitted coefficient of having embedded threads is 0.1680 ( $p=0.301$; associated odd-ratio change is $18.2 \%)$, i.e. there is no significant negative effect on completion rate by 


\begin{tabular}{|c|c|c|c|c|c|c|c|}
\hline \multicolumn{2}{|r|}{ Model } & helpful to video & useful extra & unwanted & distracting & didn't read & helpful but distracting \\
\hline \multirow{4}{*}{$\begin{array}{c}\text { Designed } \\
\text { Factors }\end{array}$} & instructor involvement $=y e s$ & $0.120(0.073)$ & $-0.023(0.071)$ & $-0.326(0.095)^{* * *}$ & $0.079(0.130)$ & $-0.248(0.075) * *$ & $0.425(0.098) * * *$ \\
\hline & thread type $=$ explanatory & $1.168(0.131)^{* * *}$ & $-0.708(0.115) * * *$ & $0.049(0.158)$ & $0.324(0.234)$ & $0.204(0.122)$ & $0.023(0.165)$ \\
\hline & timing=in the middle & $0.556(0.109) * * *$ & $-0.306(0.092) * * *$ & $-0.209(0.130)$ & $1.54(0.179) * * *$ & $-0.211(0.101) *$ & $0.611(0.128) * * *$ \\
\hline & $\begin{array}{l}\text { thread type=explanatory } \\
\& \text { timing=in the middle }\end{array}$ & $-0.783(0.171) * * *$ & $0.077(0.155)$ & $0.409(0.211)$ & $-0.499(0.299)$ & $-0.032(0.164)$ & $-0.332(0.217)$ \\
\hline \multirow{6}{*}{ Covariates } & $\log (\#$ thread-views) & $0.169(0.087)$ & $0.054(0.083)$ & $0.131(0.111)$ & $0.188(0.151)$ & $-0.042(0.088)$ & $0.173(0.116)$ \\
\hline & $\log$ (\#question-upvotes) & $0.110(0.099)$ & $-0.029(0.097)$ & $-0.149(0.130)$ & $-0.407(0.180) *$ & $0.111(0.104)$ & $-0.105(0.134)$ \\
\hline & $\log$ (\#question-replies) & $-0.207(0.085) *$ & $0.247(0.084) * *$ & $0.169(0.115)$ & $1.02(0.157) * * *$ & $-0.132(0.090)$ & $0.664(0.115) * * *$ \\
\hline & $\log$ (\#reply-upvotes) & $0.187(0.052) * * *$ & $-0.121(0.051) *$ & $-0.057(0.067)$ & $-0.206(0.092) *$ & $0.093(0.053)$ & $-0.209(0.071) * *$ \\
\hline & $\log$ (video-length) & $0.173(0.097)$ & $0.599(0.093) * * *$ & $-0.066(0.120)$ & $0.477(0.164) * *$ & $-0.243(0.096) *$ & $0.0008(0.123)$ \\
\hline & video-completion-rate & $2.26(0.750) * *$ & $1.29(0.757)$ & $3.51(1.01)^{* * *}$ & $2.52(1.33)$ & $-2.138(0.787) * *$ & $-0.702(0.999)$ \\
\hline
\end{tabular}

Table 2. The coefficients of logistic regression models for the six survey options with std. in the parentheses. Significance Code: $\mathbf{p}<0.05^{*}, \mathbf{p}<0.01 * *, \mathbf{p}<0.001 * * *$.

embedding threads in the middle of a lecture video. Instead, there is a trend suggesting that embedding threads might increase completion rate.

\begin{tabular}{|c|c|c|}
\hline Options & $\begin{array}{c}\text { \% in 21,164 } \\
\text { thread pop-ups }\end{array}$ & $\begin{array}{c}\text { \% in 15,407 } \\
\text { responses }\end{array}$ \\
\hline not-seen-survey & $19.8 \%$ & $\mathrm{n} / \mathrm{a}$ \\
\hline checked-none & $7.30 \%$ & $9.11 \%$ \\
\hline helpful-to-video & $19.5 \%$ & $26.8 \%$ \\
\hline useful-extra & $23.3 \%$ & $32.1 \%$ \\
\hline unwanted & $9.24 \%$ & $12.7 \%$ \\
\hline distracting & $7.38 \%$ & $10.1 \%$ \\
\hline didn't-read & $18.5 \%$ & $25.4 \%$ \\
\hline useful-but-interrupting & $11.9 \%$ & $16.4 \%$ \\
\hline strong-negative & $9.90 \%$ & $13.5 \%$ \\
\hline weak-negative & $4.01 \%$ & $5.51 \%$ \\
\hline mixed & $23.0 \%$ & $31.6 \%$ \\
\hline weak-positive & $2.91 \%$ & $3.99 \%$ \\
\hline strong-positive & $32.9 \%$ & $45.2 \%$ \\
\hline
\end{tabular}

Table 3. Response distribution, i.e. out of all the thread popups we displayed or survey pop-ups learners responded, what is the ratio of learner's reaction in those categories?

Learners on average saw 4.6 threads, and each thread on average was displayed to 962 learners. From Table 3, in all survey response, $45.2 \%$ of them rated strong positive, $3.99 \%$ weak positive, $13.5 \%$ strong negative and $5.51 \%$ weak negative. In summary, to a large proportion of learners, the embedded threads are rated to be useful.
Lastly, our data show that aggregating across all of the thread pop-ups, the mean CTR is $66.0 \%$, i.e. the probability of clicking through the displayed link to see more details about a discussion thread. The minimum CTR across all the threads is $37.8 \%$ and the maximum is $82.7 \%$. We believe these high CTRs suggest that learners are interested by those discussion threads which reflects the usefulness of the embedding from learners' behaviors. This is also consistent with the self-reported survey response that to a large proportion of learners, the embedded threads are useful.

$R Q 2$. We build six mixed-effect logistic regression models with the six survey options as binary responses (checking vs. not checking) respectively, modeling embedding factors and covariates as fixed effects and learners as a random intercept (shown in Table 2). The embedding factors are the three design conditions plus an interaction term between thread type and timing. The covariates are the number of thread views, the number of up-votes on the question in the thread, the number of replies for the question in the thread, the number of up-votes on the replies in the thread, length of the video in minutes and the video completion rate.

In terms of "helpful to video", instructor involvement is not a significant factor. We find a significant interaction between thread-type=explanatory and timing=in-themiddle (coef $=-0.783, p<0.001)$. Specifically, for enhancing threads, there is an increase in perceived helpfulness when they are embedded in the middle versus at the end, whereas for explanatory threads there is a significant decrease. In terms of "useful extra", we find embedding threads in the middle is associated with a decrease in being perceived as useful extra information (coef $=-0.306, p<0.001)$ and this is true for both explanatory and enhancing threads (i.e. there is no significant interaction effect). It suggests that, for 
educational goals of enhancement, presenting enhancing content in the middle might reduce its effectiveness. In addition, we find that there is an associated decrease in the probability of checking the "unwanted" option if the threads are replied by instructors than if they are not (coef $=-0.326$, $p<0.001)$. Embedding in the middle is more distracting than embedding at the end of the video $($ coef $=1.54, p<0.001)$. Learners are more likely to read the threads if the threads have instructor involvement $($ coef $=0.248, p<0.01)$ and if the threads are embedded in the middle (coef $=0.211$, $p<0.05)$.

\section{DISCUSSION}

The above results show that embedding peer discussion threads directly into lecture videos is rated to be useful by a large proportion of learners. Learners are more interested in viewing discussion threads with instructor replies (i.e., there was a lower probability of checking "didn't read" if there is instructor involvement), although this did not affect their perceived helpfulness. However, learners seem to be able to identify the relevance or quality of the thread by themselves, and hence rate threads with higher numbers of previous votes to be more helpful and less distracting as shown in Table 2.

Confirming our definition of the two types of discussion threads, we find that explanatory threads are more helpful for understanding the video itself, whereas enhancing threads are more useful as extra information. However, the interaction between thread type and timing is surprising and has important implications for future design. First, embedding enhancing threads in the middle is perceived to be more helpful to understanding the video itself, which we call the construction effect of contextual relevance. It suggests that learners tend to think of the embedded threads (even though they are enhancing content) in a narrower context and seem to create more contextual relevance between the threads and the content currently being lectured. It is not ideal for the educational goals of enhancement but also suggests that we may underestimate the usefulness of certain information in helping learners understand other related knowledge. On the other hand, contrary to the hypothesis that explanatory threads might be better being embedded in the middle to achieve the benefits of in-time help or feedback, learners perceive explanatory threads as more helpful for understanding the video itself if they are placed at the end. One benefit of this finding is reducing negative distraction because embedding at the end is perceived less distracting. Another benefit is the potential learning gain suggested by research on the delay of confusion resolution [6] which shows that delaying resolving learners' confusion might result in deeper learning later.

\section{CONCLUSION}

We propose and evaluate a reusing approach of directly embedding past high-value discussion threads into future lecture videos through an observational field study in a popular online Coursera course. We find that this approach is rated to be useful by a large proportion of learners. Our study provides guidance on three design factors of the embedding approach for future implementations, i.e. we find that instructor involvement increases learners' chance of reading the threads and reduces learners' negative reactions - but is not associated with higher perceived usefulness. Learners perceive enhancing threads embedded in the middle of videos less enhancing but more explanatory compared with at the end. Embedding explanatory threads at the end of the lecture video is less distracting and reported to be more useful in helping understand the video itself compared with embedding in the middle. Therefore, we generally propose embedding at the end of lecture videos is better both for explanatory threads which seek clarification on the lecture content and for enhancing threads which introduce useful extra information beyond the lecture. Limited by the access to Coursera platform, we leave it as future work to evaluate this approach through fully controlled experiments and thorough learning measurements.

\section{ACKNOWLEDGEMENT}

This work was supported by NSF IIS-1319382. We thank all Coursera learners who participated in our study.

\section{REFERENCES}

1. J.R. Anderson, A.T. Corbett, K.R. Koedinger, and R. Pelletier. 1995. Cognitive tutors: Lessons learned. The journal of the learning sciences, 4(2), pp.167-207.

2. D. Bargeron, A. Gupta, J. Grudin, and E. Sanocki. 1999. Annotations for streaming video on the Web: system design and usage studies. Computer Networks, 31(11), pp.1139-1153.

3. R.H. Bruning, G.J. Schraw, and R.R. Ronning. 1999. Cognitive psychology and instruction. PrenticeHall, Inc., One Lake Street, Upper Saddle River, NJ 07458 .

4. M.D. Gall. 1970. The use of questions in teaching. Review of educational research, 40(5), pp.707-721.

5. J.D. Gould, J. Conti, and T. Hovanyecz. 1983. Composing letters with a simulated listening typewriter. Communications of the ACM, 26(4), pp.295-308.

6. B. Lehman, and A. Graesser. 2015. To Resolve or not to Resolve? that is the Big Question About Confusion. In $A I E D$ (pp. 216-225). Springer International Publishing.

7. L. Vygotsky. 1978. Interaction between learning and development. Readings on the development of children, 23(3), pp.34-41. 